\title{
EDUCACIÓN EMPLEO E INGRESOS EN EL MUNICIPIO DE PASTO: UN ESTUDIO DE DATOS PANEL 2008 - 2010
}

\author{
Por: Edinson Ortiz Benavides ${ }^{1}$, Iván Freddy Erazo ${ }^{2}$, Marco Antonio Burgos ${ }^{3}$
}

\begin{abstract}
RESUMEN
La educación es la base que da soporte al crecimiento y desarrollo económico de un país y guarda una relación muy estrecha con el empleo y los ingresos de los individuos. Para muchos teóricos la educación es un bien de inversión que aumenta la probabilidad de que un individuo sea retribuido con ingresos más elevados y conseguir empleo de mayor calidad. Tanto a nivel internacional como nacional se han publicado numerosos escritos sobre el tema sin embargo, a nivel regional la literatura es escasa, razón por la cual, el presente trabajo tiene como objetivo, por una parte, contrastar el cumplimiento de esta hipótesis para el Municipio de Pasto (Nariño) y, por otra, establecer si la educación brinda mayores oportunidades de empleo para los habitantes de esta localidad. Para ello, con datos de 4.797 individuos y de 1.123 hogares, tomados en los años 2008-2010, aplicando el modelo de Mincer, se pudo establecer que un año adicional de educación aumenta aproximadamente en $5,11 \%$ el ingreso de los pastusos. Igualmente, que un año adicional de educación incrementa en 3,5\% la probabilidad de encontrar empleo en el municipio de Pasto.
\end{abstract}

Palabras clave: Educación, Empleo, Ingresos.

Clasificación JEL: 120, J30, J60.

1. Magíster en Economía Universidad de Los Andes. Especialista en Finanzas y Economista de la Universidad de Nariño. Profesor Asistente programa de Economía de la Universidad de Nariño. E-mail: edinsoneconomia@gmail.com

2. Magister en Finanzas. Universidad Mariana. Profesor Tiempo Completo Ocasional de la Universidad de Nariño. E-mail: ivanerazo59@hotmail.com.

3. Magíster en Política Económica Internacional Universidad de Belgrano, Buenos Aires. Argentina. Especialista en Finanzas y Economista de la Universidad de Nariño. Profesor Asistente programa de Economía de la Universidad de Nariño. E-mail: marcoantonioburgos@gmail.com. Agradecimiento especial a las asistentes de investigación: 


\title{
EDUCATION EMPLOYMENT AND INCOME IN THE MUNICIPALITY OF PASTO: A PANEL DATA STUDY 2008-2010
}

\author{
By: Edinson Ortiz Benavides, Iván Freddy Erazo, Marco Antonio Burgos
}

\begin{abstract}
Education is the foundation that gives support growth and economic development of a country and is very closely related to employment and income of individuals. For many theoretical education is a good investment that increases the probability that an individual is rewarded with higher incomes and get better jobs. Both international and national levels have been published numerous writings on the subject however, regional literature is scarce, which is why, this paper aims, first, to contrast the performance of this hypothesis for the Municipality Pasto (Nariño) and, secondly, to establish whether education provides greater employment opportunities for the people of this town. To do this, using data from 4,797 individuals and 1,123 households, taken in the years 2008 to 2010, applying the Mincer model, it was established that an additional year of education increases by approximately $5.11 \%$ income Pasto. Similarly, an additional year of education increases by $3.5 \%$ the probability of finding employment in the municipality of Pasto.
\end{abstract}

Keywords: Education, Employment, Income.

JEL classification: I20, J30, J60. 


\section{Introducción}

La educación es la base que le da soporte al crecimiento y desarrollo económico de un país y guarda una relación muy estrecha con el empleo y los ingresos de los individuos. Para muchos teóricos la educación es un bien de inversión que aumenta la probabilidad de que un individuo sea retribuido con ingresos más elevados y conseguir empleo de mayor calidad.

Bajo el anterior supuesto, el presente trabajo tiene como objetivo conocer los beneficios, en términos de ingresos, que un año adicional de educación tiene sobre el salario de los individuos de Pasto, así como su influencia en las probabilidades de conseguir empleo. El argumento central es que en ciudades intermedias como Pasto donde la varianza en el nivel educativo es mayor, el aporte de un año adicional de educación es menor sobre el salario, en comparación con ciudades grandes o pequeñas donde la varianza resulta menor. Para este propósito se utilizan datos panel, provenientes de la encuesta de coyuntura social utilizada en el estudio sobre Pobreza y Bienestar en Pasto a cargo del profesor Robert Wilson Ortiz, que fue aplicada de manera aleatoria a 4.797 individuos y a 1.123 hogares, pertenecientes a todos los sectores y estratos del casco urbano de este municipio.

En primera instancia, el documento analiza el entorno municipal, con el objeto de conocer el contexto socioeconómico de los hogares pastusos. Luego se aborda la revisión de la literatura relevante sobre el tema de estudio, tanto a nivel internacional como nacional, donde se destacan los trabajos de Forero y Gamboa (2007) y el de Quiñones y Rodríguez (2010), quienes estiman la relación entre educación e ingresos para diferentes escenarios nacionales y regionales. Finalmente, partiendo de la teoría del capital humano y del modelo empírico de Mincer (1974), se analizan los aportes de la educación sobre los ingresos salariales de los pastusos, así como su influencia en las probabilidades de estar empleado en un mercado laboral muy competido.

\section{Contexto: Educación, Empleo e Ingresos en Pasto ${ }^{4}$}

El municipio de Pasto se encuentra ubicado al suroccidente de Colombia, en la cordillera de los Andes, en el Nudo de los Pastos, al pie del volcán Galeras. Tiene una superficie de $1.181 \mathrm{~km}^{2}$, equivalente al 3,5\% de la superficie del departamento de Nariño. Pasto concentra el 25\% de la población del departamento de Nariño. Según proyecciones realizadas por el DANE para el año 2013, el municipio cuenta con una población de 428.890 personas, de las cuales el 82.5\% está en la zona urbana ( $2,2 \%$ de la extensión municipal) y un $17.5 \%$ en la zona rural; de ellas $51.8 \%$ son mujeres y $48.2 \%$ son hombres.

Con el análisis de las cuentas económicas del municipio de Pasto (CEDRE, 2011), se detecta que la dinámica de la economía de este municipio se ha caracterizado por ser cíclica y además volátil. Dicha volatilidad estaría advirtiendo que la economía del municipio es muy sensible a choques o perturbaciones, deriva-

4. Las cifras y datos se obtuvieron de la encuesta a 1.123 hogares pastusos, tomada en los años 2008 -2010 . 
das de las políticas económicas nacionales y regionales, y aún de las externas, dado el carácter de frontera que tiene el departamento de Nariño, del cual Pasto es su capital. No es menos importante la sensibilidad de la economía municipal a perturbaciones derivadas de fenómenos sociales presentes en Nariño, como el conflicto armado y el narcotráfico, con sus secuelas de violencia y desplazamiento forzado, cuya necesaria atención causa una presión, fundamentalmente, sobre los recursos públicos, que puede propiciar un menor dinamismo de la economía local; elementos coyunturales como el conocido caso de las pirámides o el de la amenaza volcánica, sin duda son factores que en su momento perturbaron fuertemente el comportamiento económico de la ciudad de Pasto. (ORMET, 2012).

Evidenciando la volatilidad de la economía del municipio, se observa que ésta ha tenido coyunturalmente un notable crecimiento en el año $2006(6,43 \%)$ y un crecimiento negativo en 2009 (1,33\%) sin embargo mantuvo un crecimiento promedio (simple) del $6 \%$, bastante por encima del promedio departamental $(3,8 \%)$ y del nacional $(4,28 \%)$ en el período 2006 - 2010 (Ver Figura 1).

Figura 1. Tasa de variación anual del PIB Pasto, Nariño y Colombia 2006 - 2010

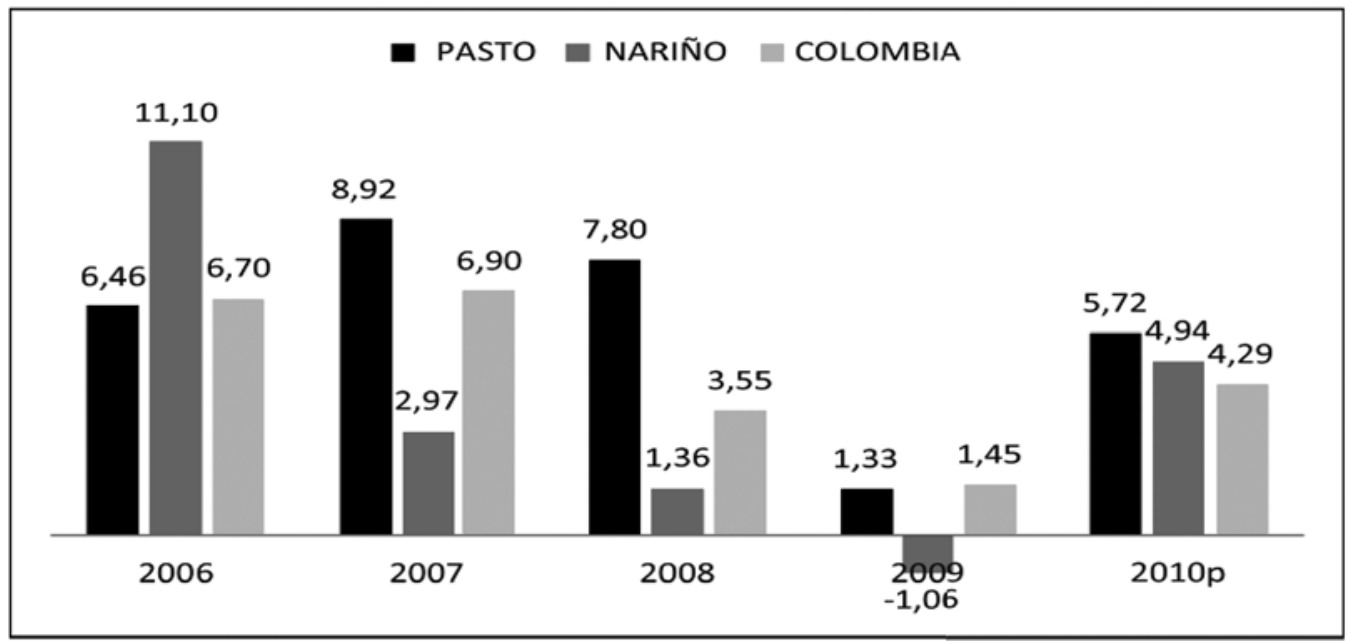

Fuente: CEDRE - Centro de Estudios de Desarrollo Regional y Empresarial (2011): Cuentas Económicas del municipio de Pasto. 2005-2010. Facultad de Ciencias Económicas y Administrativas, Universidad de Nariño. San Juan de Pasto.

En contraste con esta dinámica productiva la participación de Pasto en el PIB nacional ha sido históricamente marginal, ubicándose en promedio por debajo del 1\%. De conformidad con la disposición de datos a 2010, la participación de Pasto alcanzaba un 0,8\% en el PIB nacional y un 53,83\% en el PIB departamental. Cabe precisar además, que la participación de todo el departamento de Nariño en ese periodo en el PIB nacional fue de apenas el 1,5\%, evidenciando un apreciable rezago de la economía regional en lo que a generación de valor agregado se refiere, frente a economías de otros departamentos y ciudades, caso Antioquia y Valle del Cauca que según el DANE, son los departamentos con mayor aporte 13,4\% y 9,7\% 
respectivamente, y ciudades como Bogotá que con 25,8\% tiene el mayor aporte del país (CEDRE, 2011).

Según el Centro de Estudios de Desarrollo Regional -CEDRE-, la dinámica del sistema económico municipal y su alta participación en el PIB departamental, está determinada por las tendencias del comportamiento de las cinco ramas de actividad económica que en mayor medida contribuyen a la economía local, las cuales en el 2010 representaron el 83\% del PIB municipal, en tanto que en el aporte de las otras ocho (8) ramas restantes, tan solo fue del 17\%, situación que se ha mantenido casi constante durante los últimos años. Las cinco (5) principales actividades económicas desarrolladas por los pobladores del municipio son a saber, Servicios (28,44\%), Comercio Hoteles y Restaurantes (25,15\%), Gobierno (14,47), Industria Manufacturera (10,02\%) y Construcción (4\%).

Las anteriores actividades económicas dejan, en promedio para el periodo 2008 - 2010, ingresos mensuales a precios corrientes de \$ 885,984 según la encuesta aplicada a los 1.123 hogares. Si se mide por estratos se tiene que en el estrato I la media es de \$ 436.765, en el II de \$ 554.662, en el III \$ 1.074.579, en el IV 1.711.833, en el V \$ 1.719.722 y en el VI \$ 5.000.000, lo que evidencia una gran brecha entre ellos. Los datos advierten que el 65\% de los encuestados manifestó que sus ingresos no son suficientes para cubrir sus gastos mensuales, y aspiran que en promedio éste se incremente en un 64\%, siendo los del estrato I, los más inconformes con el ingreso recibido.

Por otro lado, en materia de empleo, la desocupación en Pasto con un 15\% se ubica por encima del promedio nacional (10,8\%) como una de las ciudades con mayor desempleo del país, evidenciando una mayor oferta laboral con respecto a la demanda existente. El mercado laboral local se caracteriza por tener una tipología de desempleo cíclica, es decir, que el desempleo es explicado fundamentalmente por una reducción de la demanda agregada, concretamente de alguno o varios de sus componentes, como las exportaciones, el consumo, la inversión o el gasto público.

Dada la gran dinámica del sector comercio, frente a la débil de la industria manufacturera, analizada en el capítulo sobre estructura económica, se podría plantear que buena parte del comportamiento cíclico del desempleo sería explicado por la contracción que periódicamente sufre la actividad comercial, ésta derivada, a su vez, por una reducción en el consumo (CEDRE, 2011).

En materia educativa, para 2013, según datos de la Secretaría de Educación, el 63,39\% de la población residente en Pasto había alcanzado el nivel de transición, 87,1\% la básica primaria, el 71,8\% la básica secundaria, el 40,98\% la media técnica y el $81,9 \%$ había realizado estudios profesionales y un 3,4\% estudios a nivel de postgrado. La tasa de analfabetismo entre las personas de 15 o más años era de $6,42 \%$, muy por debajo del promedio del departamento $(11,5 \%)$ y del país $(6,7 \%)$.

Conforme lo anterior, se pone de manifiesto la necesidad de implementar, por parte de las autoridades locales y regionales, políticas económicas con el fin de reducir el desempleo, mejorar los ingresos y la calidad educativa, que posibiliten un mejoramiento generalizado de las condiciones de vida en la ciudad, y un mayor aporte departamental a la economía nacional. 


\section{Revisión de la Literatura}

La relación entre educación, empleo e ingresos es un tema de amplio y constante debate. Los retornos o rentabilidad de la educación miden frecuentemente la relación entre educación e ingresos. En el ámbito internacional, aparte de los estudios pioneros de Schultz, Becker y Mincer destaca el trabajo de Blaug (1970), quien examina tres explicaciones alternativas para la relación positiva entre educación e ingreso: una explicación económica (la gente mejor educada gana más porque la educación provee de habilidades que son escasas en el individuo), una explicación social (la educación difunde valores sociales que son valorados) y una explicación psicológica (la educación selecciona a la gente de acuerdo con sus habilidades, y la gente más hábil percibe mayores ingresos). Otros trabajos realizados en países desarrollados, que estudian la inversión en capital humano y su relación con el ciclo de vida del ingreso, son los de Haley (1976), Johnson (1980), Kenny, Lee, Maddala y Trost (1979), y Garen (1984), que estiman el rendimiento de la escolaridad con base en modelos de selección por sesgo. A su vez, Salas (2004) estima el rendimiento de la educación usando la ecuación estándar de Mincer y cuestiona su utilidad para el caso de la educación superior cuando ésta es una variable endógena, pues al considerarla exógena, la estimación sesga hacia abajo la tasa de rentabilidad en aproximadamente cinco puntos porcentuales.

De modo similar, en varios estudios que se han realizado para países en desarrollo se ha podido establecer que existe una estrecha relación entre la educación y los ingresos aunque, también, se han encontrado evidencias que contraponen o limitan el modelo teórico por ejemplo, Raymond y Manaloto (1990) estiman el rendimiento de la escolaridad en China, con base en el modelo de Mincer; y obtienen que hay rendimientos decrecientes en la educación y en la experiencia, y que éstos son sustitutos cercanos. Lau, Jamison, Liu y Rivkin (1993) estudiaron la relación entre educación y crecimiento económico en Brasil, y encontraron que el capital humano ha tenido una influencia positiva, aunque no tan importante como el progreso tecnológico. Rojas, Angulo y Velásquez en el año 2000 para el caso de México, estiman la tasa interna de retorno de la inversión en capital humano incorporando el costo de oportunidad que se origina por salario no percibido durante el tiempo de estudios, encontrando que la tasa interna de retorno estimada es, por lo tanto, menor a la que se obtiene siguiendo la metodología original de Mincer (1974).

Para el caso colombiano, los primeros trabajos se remontan a la década del sesenta, con los estudios de Schultz (1968) y Selowsky (1968) quienes encuentran tasas de retornos sociales de la educación decrecientes con el nivel de escolaridad, mientras que Kugler (1975) estima que la tasa de retorno para la educación primaria es inferior a la de la secundaria y no encuentra diferencias significativas entre secundaria y superior.

Por su parte, González (1982), utilizando datos de 209 trabajadores bogotanos de 87 pequeñas empresas, analiza los retornos de la educación en diferentes sectores y su relación con la productividad de la pequeña industria. En otro estudio, Psacharopoulos y Vélez (1988) estimaron un modelo sobre escolaridad, habilidad e ingresos en Colombia y encontraron que el rendimiento obtenido justifica la inversión en educación. 
Alternativamente, Tenjo (1993a) para el periodo 1976-1989 reconoce la existencia de un sesgo en las estimaciones del retorno de la educación por falta de información sobre la habilidad del trabajador o su ancestro socioeconómico. En otro artículo, para el mismo período, este autor (Tenjo, 1993b) analiza la evolución de los retornos de la educación y encuentra una reducción en los mismos como resultado de aspectos como la expansión del sistema educativo del país en los años anteriores, el aumento de la participación femenina en el mercado laboral y el incremento en su nivel de escolaridad.

Otros trabajos que se han realizado en el país está el de Perfetti (1996), donde encuentra que las diferencias salariales entre los años 1984 y 1994 se explican en un $16 \%$ y $11 \%$ por los distintos grados de acumulación de capital humano, respectivamente. Por su parte, Núñez y Sánchez (1998) afirman que la tasa de retorno de la educación ha disminuido desde comienzos de la década de 1980. Castellar y Uribe (2001), siguiendo la teoría del capital humano de Becker (1964), identifican los retornos de la educación en Colombia entre los años 60 y 70. Forero y Gamboa (2007) estiman la tasa de retorno de la educación de la población asalariada de Bogotá para 1997 y 2003 teniendo como referencia la teoría del capital humano. Usando datos de la encuesta de calidad de vida encuentran que la rentabilidad de la educación es positiva y significativa, aunque hubo una reducción entre los dos periodos de análisis.

Teniendo en cuenta la parte metodológica, para corregir el sesgo por falta de información sobre la habilidad o condiciones socioeconómicas del individuo, Casas et al (2003) proponen solucionarlo con variables instrumentales y concluyen que los retornos no difieren mucho cuando se controla por habilidad y que al usar una variable de caracterización socioeconómica como instrumento se puede incurrir en una sobreestimación de los retornos. Zárate (2003), utilizando regresión por cuartiles para el periodo 1991-2000 con base en la ENH, encuentra que los retornos de la escolaridad son superiores en los cuartiles de ingresos más altos, que las fluctuaciones son mayores en los cuartiles inferiores y que en promedio la variabilidad en los cuartiles intermedios es mayor que en los extremos además, en los cuartiles inferiores se evidencia un deterioro en los retornos de la educación entre 1991 y 2000. Barrero et al (2004) utilizan la ENH de 2000 y obtienen el retorno de la educación corrigiendo el sesgo de selectividad sin suponer que la elasticidad horas trabajo del ingreso es unitaria. Concluyen que los niveles de escolaridad son un determinante significativo del ingreso percibido y que la hipótesis sobre la elasticidad debe rechazarse.

Siguiendo con el aspecto metodológico, Mora (2003) analiza el efecto screening en el mercado laboral de siete ciudades a partir de la ENH del año 2000 y encuentra evidencias para afirmar que los diplomas sí generan un ingreso adicional en los trabajadores. Prada (2006) estudia la dinámica de los retornos de la educación en Colombia entre 1985 y 2000, para ello utiliza la ENH de septiembre de cada año y realiza la regresión de salarios con variables spline, y también por el método de Regresión por Percentiles (RP). Los resultados son consistentes con otros documentos ya que los retornos de la educación universitaria son más altos igualmente, la evidencia de la RP muestra un comportamiento cíclico del salario real, y que los 
beneficios de la educación postuniversitaria son más altos pero más sensibles al ciclo económico.

En materia de género, Velásquez (2001) analiza las diferencias salariales por género utilizando la descomposición de Oaxaca y corrige el sesgo de selección con la metodología de Heckman, concluye que las diferencias en el rendimiento de los individuos explican la brecha salarial por género. Estudios posteriores como el de Tenjo y Bernat (2002) concluyen que los salarios de hombres y mujeres se determinan a través de mecanismos distintos y que las diferencias en ingresos se han reducido. Arias y Chaves (2002) utilizando el procedimiento de Heckman obtienen las ecuaciones adel modelo Spline por género, con datos de corte transversal para 1991, 1999 y 2000 de la ENH; encuentran que los retornos a la educación son mayores en las mujeres aunque el diferencial se ha reducido, concluyen que tanto en hombres como en mujeres la tasa interna de retorno de la educación se ha deteriorado en los últimos años.

En síntesis, la evaluación de la relación entre educación e ingresos en Colombia no permite establecer una tendencia clara en temas como género, nivel educativo y cohortes. Sin embargo, las metodologías aplicadas han ido superando los errores de especificación inicialmente encontrados, e incluyen estimaciones por mínimos cuadrados, panel de datos, regresión por cuantiles, Heckman y descomposición de Oaxaca, que ha permitido avanzar en el análisis.

Queda evidenciada la relación causa-efecto entre la educación y el nivel de ingresos o crecimiento económico; empero, esta relación depende de la correcta articulación entre la formación educativa y las exigencias del mercado de trabajo. De tal modo, el mercado laboral se convierte en el puente que une a la economía con la educación. Entendiendo que, tanto la educación formal y para el trabajo, como la formación dentro de la empresa, tienen un impacto positivo en el incremento de la productividad.

Aun cuando, la educación aporta al crecimiento de las economías esta propiedad no es la única ni la más importante. Gracias a la educación se puede aspirar a una distribución más equitativa de la riqueza nacional. Se considera que la inversión en educación es fundamental para el escalamiento social porque aporta más oportunidades de emplearse a las personas y también se le brinda mejores condiciones de empleo.

En la hipótesis de la selección Arrow (1972) sugiere que la educación sirve de mecanismo para distinguir entre los empleados más deseables y los menos deseables. De esto se desprende que la educación es un medio para escoger personas y asignarles un empleo; y que en sí, un empleo de mayor productividad es al que le corresponden salarios mayores (Carnoy, 1977). Entonces, la correcta correspondencia entre formación educativa y las exigencias del mercado laboral permiten que las personas adquieran empleos con mejores condiciones. Este fue el objeto de estudio de los autores Eijs y Heijke (1996); quienes determinaron que los empleadores buscarán trabajadores cuyas habilidades estén alineadas con los requerimientos para el empleo y que sean capaces de lograr la productividad óptima (López, 2009). 
Para el caso del departamento de Nariño, Quiñones y Rodríguez (2010), que toman como referente la teoría del capital humano, examinan las diferencias regionales como indicador de las variaciones en los ingresos de los individuos pertenecientes a ellas. En su investigación utilizan estimaciones de mínimos cuadrados ordinarios y la descomposición de Oaxaca-Blinder, corrigiendo el sesgo de selección por el método de Heckman, aplicada a la Encuesta Continua de Hogares en el segundo trimestre del 2006 para las trece áreas metropolitanas, hallando un 40\% de diferencia en la remuneración de los ocupados especializados en Medellín con respecto a los de Pasto. Cuando se incluye el efecto educación, la diferencia se reduce a $20 \%$ así mismo, encuentran que Pasto pertenece al tercero de los grupos identificado por ellos, el cual corresponde a las regiones del país con menor retribución de la educación sobre el salario.

En el caso del municipio de Pasto, se destacan los trabajos de Riascos y Erazo (2010) quienes, en un estudio de corte transversal, encuentran que un año adicional de educación superior incrementa en 7,9\% los ingresos laborales. Asimismo, aplicando la metodología propuesta por Halvorsen y Palmquist establecieron que los retornos en formación de postgrado (54,7\%) fueron extraordinariamente superiores. La hipótesis central derivada de los resultados sugiere que, en cuanto a absorción de ingresos existe un potencial desplazamiento de trabajadores con formación de pregrado, por fuerza laboral con educación posterior. Luego, Riascos en el 2011 en un estudio sobre situación laboral de la mujer en Pasto, plantea que, en la actualidad, los tres principales problemas que enfrenta la mujer profesional en el mercado laboral descansan en el elevado subempleo $13,56 \%$, en particular por insuficiencia de horas 10,55\%, el desempleo 8,16\% y la persistente brecha salarial evidenciada ya a nivel nacional. Mientras que en el Municipio un profesional devenga 2,915 Salarios Mínimos Legales Mensuales Vigentes (SMMLV) una profesional percibe 2,447 SMMLV, esto es 16,1\% menos que el hombre. En el 2013 el CEDRE en convenio con la Red de Observatorios Regionales del Mercado de Trabajo - ORMET publican el documento titulado "Brechas de género en el mercado de trabajo de Pasto (2007-2012)" y encuentran no solo para el sector de profesionales, sino para la población en edad de trabajar en general, que la brecha salarial para las mujeres es desfavorable entre un $25 \%$ y $37 \%$ para el periodo de estudio, en relación con los trabajadores masculinos.

\section{MARCO TEÓRICO}

Hay tres maneras distintas de ver la educación (Johnson, 1975): a) Como un bien de consumo, el cual produce satisfacción o utilidad sin alterar la productividad del individuo. b) Como un filtro, el cual permite identificar a los individuos con las más altas habilidades innatas o características personales, las cuales los hacen ser más productivos para obtener mayores ingresos salariales. Por lo tanto, la educación no afecta la productividad del individuo, pero sí le permite enviar una señal sobre sus características innatas. c) Como capital humano, el cual analiza el gasto en educación como una forma de inversión, la cual hace a los individuos más productivos y por lo tanto genera un salario esperado mayor en el futuro. 
La idea original de considerar a la educación como una forma de inversión, que posteriormente termina en la teoría del Capital Humano (Schultz, Becker y Mincer) proviene de Adam Smith, en su libro "Investigación sobre la Naturaleza y Causas de la Riqueza de las Naciones", intuye la importancia de la educación sobre la determinación de los salarios corrientes y futuros entre distintos tipos de trabajadores. De acuerdo con Smith, "los salarios del trabajo varían con la facilidad y baratura o bien con las dificultades y elevados costos del aprendizaje”. En este libro también se encuentran ideas innovadoras como el rendimiento por invertir en escolaridad, la influencia de la habilidad innata y adquirida sobre la productividad, así como el factor tiempo para explicar el por qué en edades tempranas se tiende a invertir más en educación que en edades avanzadas.

Pese a que Smith ya había establecido las bases de la teoría del Capital Humano, no fue sino hasta mediados de los cincuentas y principios de los sesentas del siglo pasado que se desarrollaron modelos en relación a sus ideas que pudieran explicar las brechas salariales entre trabajadores y el desempleo. Como respuesta, por primera vez se crearon modelos que consideraban a la inversión en educación como una decisión que los individuos toman con fines que pemitirían incrementar el ingreso futuro, la utilidad y el bienestar de las personas. Es decir, los supuestos de la economía neoclásica de racionalidad en la toma de decisiones y de individuos maximizadores de beneficios bajo ciertas condiciones fue posible introducirlo a la economía de la educación.

En 1961 el economista Theodore W. Schultz desarrolló el concepto de capital humano, concepto que se refiere a todas aquellas capacidades humanas desarrolladas por la educación, que pueden usarse de manera productiva y que por lo tanto poseen un valor de mercado intrínseco. Esta teoría o enfoque del capital humano explica la relación entre ingresos y educación como resultado de la mayor productividad que adquieren las personas que poseen una mayor educación, es decir, puede atribuirse a la educación, el desarrollo y la formación de habilidades y destrezas, que son redituadas por el mercado a través de un mayor ingreso.

Por otra parte, siguiendo el enfoque de Becker (1964), la decisión de asistir a la escuela puede ser vista como una inversión donde el individuo sustituye recursos presentes (costos directos y costos de oportunidad) por futuras ganancias. La inversión en educación implica que la percepción de ingresos se difiere, pero las ganancias posteriores (medidas a partir del diferencial de ingreso obtenido por individuos con diferentes niveles o grados de escolaridad) superarán los costos iniciales generando incentivos para la acumulación de capital humano. Se supone que, en equilibrio, los individuos invertirán en educación hasta que el retorno marginal de los ingresos futuros descontados iguale a los costos marginales de la educación. Aquí, la tasa de descuento a la cual los beneficios netos de la inversión se hacen cero corresponde precisamente a la tasa interna de retorno de la educación.

Ahora bien, el primer costo que enfrenta un individuo al realizar un grado adicional de estudios es el costo de oportunidad por salario no percibido. La incorporación de este costo hace que la tasa de retorno de la educación se reduzca. Para calcular esta tasa de retorno se combina el modelo de Mincer (1974) con el modelo de Rosen (1977), planteándose los siguientes supuestos básicos: 
a) Los individuos consideran la educación como una inversión en capital humano.

b) El costo financiero para el individuo de adquirir educación es cero $^{5}$, lo que implica que estos costos no son pagados por la persona que realiza los estudios. Por lo tanto, sólo se enfrenta el costo de oportunidad, representado por los ingresos no percibidos con el nivel de escolaridad actual ${ }^{6}$.

c) Durante el tiempo de estudios no se realizan actividades remunerativas.

d) El ingreso por salario se determina según el nivel de educación o estudios adquiridos y otras variables explicativas.

e) El individuo tiene dos cursos de acción en $t$ : trabajar y obtener un salario $X_{t}$ dado su nivel de escolaridad, o continuar estudiando para obtener un salario real al concluir un nivel de estudios superiores ${ }^{7}$. La información para estos escenarios es obtenida de la experiencia de otros individuos, los cuales ya siguieron uno de los dos cursos.

La información de realizar o no estudios adicionales depende de los rendimientos en exceso en un momento $t$, definidos como:

$$
Z_{t}=Y_{t}-X_{t}
$$

Obteniendo la sumatoria de todos los flujos de efectivo y descontando a valor presente se obtiene:

$$
V_{t}=\sum_{t=u}^{n} \frac{Z_{t}}{(1+r)^{t}}(2) \text { o su versión continúa: } V_{t}=\int_{t=u}^{n} Y_{t} e^{-r t} d_{t}-\int_{t=u}^{n} X_{t} e^{-r t} d_{t}(3)
$$

Donde $V_{t}$ es el valor presente neto, $t$ es el tiempo, $r$ es la tasa de descuento temporal, $u$ es el último año de estudios y $n$ es la edad de retiro. Es económicamente conveniente continuar con los estudios si $V_{t}>0$. La principal dificultad al realizar un análisis de valor presente es determinar qué tasa de descuento temporal debe emplearse. Para obviar este problema, en vez de calcular el valor presente neto se calcula la tasa interna de retorno $(R)$, esto es, la tasa de descuento temporal a la cual $V_{t}=0$, entonces:

$$
0=\sum_{t=0}^{n}\left[\left(\frac{Y_{t}}{(1+R)^{t}}\right)-\left(\frac{X_{t}}{(1+R)^{t}}\right)\right]
$$

5. Los costos financieros incluyen colegiaturas, materiales didácticos, manutención, etc. A estos costos se le atribuye un valor de cero, para simplicidad del modelo, y bajo un contexto de educación gratuita, cuyo camino han empezado a transitar muchos países latinoamericanos, incluido Colombia.

6. Otro costo en el que se puede incurrir es el desgaste físico e intelectual que varía de acuerdo con el tipo de preparación y aptitudes del individuo

7. De acuerdo con el supuesto c), para los años de instrucción, durante el periodo $u$ - $t$ (donde $u$ es el último año en la escuela). Se tiene que: $t=0$ o $\Sigma Y_{t}=0$ 
El primer término del lado derecho de la ecuación (4), indica el valor presente del ingreso esperado de un individuo que decide continuar sus estudios por un periodo adicional igual a $u$. El segundo término, indica el valor presente del ingreso de un individuo que decide ir inmediatamente al mercado laboral en $t$ con su nivel de escolaridad presente. Si el primer término es mayor que el segundo, existe un incentivo económico para obtener mayores niveles de escolaridad. Para calcular la tasa interna de retorno $R$ que resuelve la ecuación (4) se utiliza un método iterativo de aproximación (Ross, 1995). A mayor tasa interna de retorno, mayor es el incentivo económico que la continuación de estudios provee. Por lo tanto, es de interés calcular el comportamiento de la tasa interna de retorno conforme se avanza en la escolaridad, a fin de observar si el incentivo económico para que un individuo continúe estudiando es suficiente.

Si el individuo tiene una función de utilidad intertemporal de la forma:

$$
U=\int_{t}^{n} e^{-\rho t} U(\cdot) d_{t}(5)
$$

Donde $\rho$ indica la tasa subjetiva de descuento temporal de acuerdo con sus preferencias intertemporales, la cual refleja su impaciencia en cuanto a consumo y otras actividades que se ven influenciadas por la decisión de percibir un ingreso menor en determinado momento o esperar por un ingreso mayor en el futuro, entonces la condición necesaria para que este individuo continúe estudiando se da cuando $\rho<R$.

Una $R$ muy baja (o un $\rho$ muy alto) hace menos probable que un individuo decida la conveniencia de continuar sus estudios. Un segundo costo que debería contemplarse, relajando el supuesto $b$, es el costo financiero. La incorporación del costo financiero reduciría aún más la tasa interna de retorno. Sin embargo, la incorporación de este costo es compleja, pues se requiere de información sobre todos los costos en que un individuo incurre al estudiar.

\section{El modelo empírico}

Para la implementación empírica del anterior modelo teórico, se estima econométricamente una función de ingresos para recuperar el valor de un parámetro ( $r$ ) que represente el cambio en la remuneración derivado de un incremento en el nivel de educación del individuo. En este caso, la herramienta empírica utilizada en la mayoría de estos trabajos ha sido la ecuación minceriana de ingresos (Mincer, 1974), a través de la cual se estima el impacto de un año adicional de estudios en las rentas laborales de los individuos (Freire y Tejeiro, 2011).

La ecuación tradicional de Mincer, estima por mínimos cuadrados ordinarios (MCO) un modelo semilogarítmico, usando como variable dependiente el logaritmo de los ingresos y como variables independientes los años de educación, la experiencia laboral y el cuadrado de ésta, más controles adicionales. Los datos utilizados para su estimación provienen tradicionalmente de datos transversales, pero en esta investigación se utilizarán datos panel:

La ecuación sería la siguiente: 


$$
\operatorname{Ln}(\text { Salario })_{i, t}=\gamma_{0}+\gamma_{1} \text { Educación }_{i, t}+\gamma_{2} \text { Experiencia }_{i, t}+\gamma_{3} \text { Experiencia }_{i, t}^{2}+\gamma_{4} \text { Controles }_{i, t}+\varepsilon_{i, t}
$$

donde:

Salario: Ingresos mensuales del individuo

Educación: El más alto número de años de educación alcanzado por el individuo Experiencia: Años de experiencia laboral

Experiencia ${ }^{2}$ : Años de experiencia laboral al cuadrado

Controles: Vector de variables adicionales que condicionan el salario del individuo $\left(0, \sigma_{\varepsilon}^{2}\right)$

$\varepsilon:$ es el término de perturbación aleatoria que se distribuye según una Normal

$\boldsymbol{i}, \boldsymbol{t}:$ Representa los subíndices para el individuo $\boldsymbol{i}$, en el tiempo $\boldsymbol{t}$.

La función de ingresos de Mincer postula el paralelismo de los perfiles del logaritmo del ingreso con respecto a los distintos niveles de educación (Sapelli, 2003), si se supone cumplidas las hipótesis bajo las que se desarrolla la función de Mincer ${ }^{8}$ el valor del coeficiente de los años de educación formal se interpreta como la tasa de rendimiento media de un año adicional de estudio poseído por los trabajadores. Por otro lado, y teniendo en cuenta la teoría de los perfiles de edadingresos (conforme aumenta la experiencia, los ingresos individuales aumentan, pero cada año de experiencia tiene un efecto sobre los ingresos menor que el anterior), se espera que al ser la función cóncava con relación a la experiencia, la estimación de $\gamma_{2}$ sea positiva y la de $\gamma_{3}$ sea negativa.

La popularidad de esta ecuación se ha hecho extensible en múltiples ámbitos debido a su facilidad de aplicación y a su gran capacidad para generar resultados razonables para distintos conjuntos de datos (diferentes países, instantes de tiempo, etc.). Es preciso comentar que la anterior ecuación se construye bajo los supuestos del modelo neoclásico del funcionamiento del mercado de trabajo: las empresas conocen la productividad marginal de cada trabajador y el proceso competitivo hace que los salarios dependan de dicho nivel de productividad (Freire y Tejeiro, 2011).

Con el tiempo las aportaciones científicas demostraron que en la medición del rendimiento de la educación estimado por MCO (ecuación minceriana de ingresos) existían varios sesgos, Griliches (1977), identificó los siguientes:

a) Existencia de determinadas variables omitidas en la ecuación de Mincer como la habilidad del individuo. Si el término de perturbación incluye entre otros

8. a) Los costes de la inversión en educación son, únicamente, los costes de oportunidad (los ingresos que dejan de percibirse).

b) el tiempo que un individuo permanece en el mercado laboral es independiente del nivel de estudios alcanzado, y se supone que su permanencia en el mismo es continua.

c) Los individuos comienzan a trabajar inmediatamente después de finalizar sus estudios. 
elementos la habilidad del individuo, y se cumple que las personas con mayor habilidad son las que deciden elegir el mayor nivel educativo, esto provocaría estimaciones inconsistentes, pues la perturbación aleatoria y el nivel educativo (parámetro Educación) estarían correlacionados y esto daría, como resultado, que se sobreestimase el rendimiento de la educación.

b) Medición incorrecta de la cantidad de educación. La ecuación de Mincer implica que existe una única tasa de rendimiento de la educación, sin embargo, tanto la teoría como la evidencia empírica lo contradicen, sugiriendo representar la escolaridad en forma más desagregada y flexible, con el objetivo de recoger de una forma más adecuada los retornos al proceso de inversión en educación (Sapelli, 2009). El no recoger correctamente el nivel educativo estaría provocando una infraestimación en el verdadero rendimiento.

c) Tratamiento de la educación como una variable exógena. Diversos estudios han demostrado que la variable educación puede no ser exógena estando afectada por diferentes variables como puede ser los costes directos de la educación, los costes de oportunidad, los ingresos futuros, las imperfecciones del mercado de capitales o incluso la importancia del motivo consumo. El no considerar la endogeneidad de la educación producirá que las estimaciones realizadas por la técnica de MCO proporcione estimadores sesgados e inconsistentes del rendimiento de la educación. Si se demuestra que la variable educación es endógena, el método más apropiado para calcular sus rendimientos sería estimarlo por "mínimos cuadrados en dos etapas" (MC2E). En este método la variable que causa la autocorrelación se elimina sustituyéndola por una variable instrumental.

Además de los sesgos recogidos por Griliches (1977), existen otros dos sesgos de importancia, el primero tiene que ver con el uso de información de corte transversal para construir cohortes artificiales, en vez de utilizar cohortes efectivas a lo largo del tiempo. Esta forma de trabajar que está obviamente determinada por la disponibilidad de información, asume un comportamiento de las condiciones económicas muy estable y un mecanismo de generación de expectativas, asociado al proceso de inversión en capital humano, estático.

El segundo consiste en la selección muestral, el problema derivado de este sesgo surge de sólo poder observar las rentas salariales de aquellos individuos ocupados. Aparece principalmente, cuando ciertos individuos se encuentran sistemáticamente más presentes en indeterminado grupo frente a otro (Heckman, 1979). Para corregir el sesgo de selección se recomienda utilizar el procedimiento de dos etapas propuesto por Heckman que consiste en especificar la probabilidad que tiene el individuo de estar ocupado, a partir de un modelo probit (Freire y Tejeiro, 2011).

\section{Los datos}

Los datos de Panel utilizados en el modelo empírico provienen de la encuesta aplicada a 4.797 individuos en el Municipio de Pasto durante los años 2008 - 2010 (1.631 en 2008, 1.504 en 2009 y 1.662 en 2010), correspondiente a 1.123 hogares durante este mismo periodo (381 en 2008, 353 en 2009 y 389 en 2010). La encuesta 
recoge información de individuos de todos los estratos sociales, áreas geográficas, nivel de estudios y de salarios del casco urbano municipal, por lo tanto se considera que la muestra es representativa de la población ocupada y no ocupada de esta localidad. Se advierte que en principio el número de años del panel puede resultar limitado al ofrecer pocos grados de libertad, ante lo cual se debe ser precavido en las inferencias.

Las estadísticas descriptivas se detallan a continuación:

Tabla 1. Estadística Descriptiva

\begin{tabular}{|l|l|r|c|c|r|}
\hline \multicolumn{1}{|c|}{ Variable } & \multicolumn{1}{|c|}{ Definición } & \multicolumn{1}{c|}{ Media } & Desviación & Mínimo & Máximo \\
\hline Salario & Salario Mensual en Pesos & 826458.7 & 889152.7 & 0 & 8000000 \\
\hline Educación & Educación en Años & 9.616477 & 4.710534 & 0 & 18 \\
\hline Experiencia & Experiencia en Años & 20.262380 & 19.955610 & 0 & 86 \\
\hline Ocupado & Dummy para ocupados & 0.34605 & 0.4757588 & 0 & 1 \\
\hline Casado & Dummy para casados & 0.358349 & 0.4795654 & 0 & 1 \\
\hline Pasto & Dummy para oriundos de Pasto & 0.784657 & 0.4111029 & 0 & 1 \\
\hline Mujer & Dummy para mujeres & 0.561601 & 0.4962425 & 0 & 1 \\
\hline
\end{tabular}

Fuente: Esta Investigación

Los datos indican una alta variabilidad sobre todo en el salario, la educación y los años de experiencia, evidenciando heterogeneidad de los mismos. El promedio general de ingresos es de \$826.458,7 con una desviación de \$889.152,5, tomando valores entre \$0 y \$8.000.000. El promedio de educación en la población es de 9,6 años con una desviación de 4,7 años, tomando valores entre 0 y 18 años de educación. Asimismo, la experiencia laboral registra un promedio de 20,3 años con una desviación de 20 años, tomando valores entre 0 y 86 años.

Cuando estos promedios se analizan por género y procedencia, los resultados ganan mayor interés, veamos:

Tabla 2. Estadísticas Descriptivas por Género

\begin{tabular}{|l|r|r|r|r|r|r|r|r|}
\hline \multirow{2}{*}{ Variable } & \multicolumn{4}{|c|}{ MUJERES } & \multicolumn{3}{c|}{ HOMBRES } & \\
\cline { 2 - 10 } & Media & \multicolumn{1}{|c|}{ Desviación } & Mínimo & Máximo & Media & Desviación & Mínimo & Máximo \\
\hline Salario & 768025.7 & 879666.7 & 0 & 7500000 & 882913.3 & 895134.2 & 0 & 8000000 \\
\hline Educación & 9.742411 & 4.704961 & 0 & 18 & 9.465068 & 4.713999 & 0 & 18 \\
\hline Experiencia & 20.405990 & 19.882470 & 0 & 86 & 20.089770 & 20.047000 & 0 & \\
\hline
\end{tabular}

Fuente: Esta Investigación

Como se observa, los hombres del municipio de Pasto tienen mayores ingresos que las mujeres, pero éstas registran mayor experiencia laboral y niveles de edu- 
cación promedio, ratificando un problema de brecha laboral señalado en investigaciones locales, nacionales e internacionales. De acuerdo con Becker (1985), las diferencias en las remuneraciones por tipo de sexo se explican por un factor de discriminación en el salario entre hombres y mujeres, que puede darse incluso cuando la participación en el mercado laboral sea la misma; es decir, a igual inversión en capital humano, el retorno de las mujeres será menor que el de los hombres. Una explicación derivada de este diferencial se debe a que las mujeres deben hacerse cargo del cuidado del hogar, lo cual la limita para cargos con una alta carga horaria y disponibilidad para viajar. De esto sugiere que las diferencias en productividad determinan la división sexual del trabajo, argumentando ventajas comparativas entre familias y mercados de actividad (ORMET, 2013). Esta diferencia equivale casi a la quinta parte de un Salario Mínimo Mensual en el país.

También se analiza que las personas oriundas de Pasto poseen menores ingresos, años de educación y experiencia promedios que las personas que no lo son. En este caso no se aprecia un efecto discriminatorio. Se observa por ejemplo una diferencia de casi $\$ 220.000$ mensuales. (Ver tabla 3).

Tabla 3. Estadísticas Descriptivas por Lugar de Procedencia

\begin{tabular}{|l|c|r|c|r|r|r|r|r|}
\hline \multirow{2}{*}{ Variable } & \multicolumn{4}{|c|}{ PASTUSOS } & \multicolumn{4}{c|}{ NO PASTUSOS } \\
\cline { 2 - 9 } & \multicolumn{1}{|c|}{ Media } & Desviación & Mínimo & Máximo & Media & Desviación & Mínimo & Máximo \\
\hline Salario & 784711 & 842362.9 & 0 & 7500000 & 882913.3 & 1048859 & 0 & 8000000 \\
\hline Educación & 9.548805 & 4.669182 & 0 & 18 & 9.465068 & 4.888253 & 0 & 18 \\
\hline Experiencia & 19.782900 & 19.723490 & 0 & 86 & 20.089770 & 20.871560 & 0 & 86 \\
\hline
\end{tabular}

Fuente: Esta Investigación

Conforme los datos de la investigación, tanto la educación como la experiencia muestran relaciones simples, en la misma dirección de estudios nacionales e internacionales. El salario guarda una relación directa con los años de educación y con la experiencia laboral, es decir, mayores años tanto de educación como de experiencia laboral generan un incremento positivo en el salario mensual de las personas. Sin embargo, este tipo de análisis gráfico de Mínimos Cuadrados Ordinarios (MCO) resulta insuficiente, entre otras razones, debido a la falta de controles y la posibilidad de sesgo (ver figura 1).

\section{Resultados}

Pruebas econométricas (Breusch Pagan) a los datos evidenciaron con un 99\% de confianza la presencia de heterogeneidad no observada en el término de error, lo cual implica la necesidad de usar alguna de las metodologías que tengan en cuenta la existencia de efectos constantes en el tiempo. Por ello, se hicieron regresiones mediante efectos aleatorios (EA) y efectos fijos (EF) y al realizar la prueba de Hausman, se pudo establecer con un 99\% de confianza una fuerte evidencia estadística de endogeneidad. En este sentido, el estimador adecuado es el de Efectos Fijos cuyos estimadores son eficientes e insesgados (Rosales, et. al., 2013). 
Figura 1. Relación Salario vs. Educación y Experiencia, Pasto 2008 - 2010.
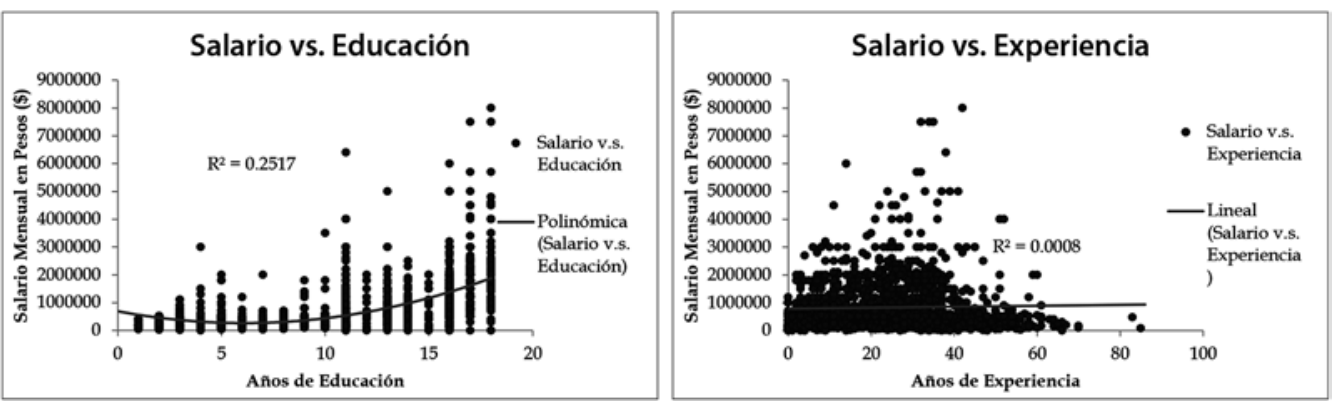

Fuente: Esta Investigación

Los datos corroboraron la relación positiva entre el salario percibido por una persona y sus años de educación y experiencia. Estos resultados se obtienen a un nivel del 99\% de confianza y resultan persistentes a diferentes controles (ver tabla 4).

Tabla 4. Educación e Ingresos en Pasto, 2008 - 2010

\begin{tabular}{|c|c|c|c|c|c|}
\hline \multirow{2}{*}{ VARIABLES } & \multicolumn{5}{|c|}{ Logaritmo Natural del Salario - Efectos Fijos } \\
\hline & (1) & (2) & (3) & (4) & (5) \\
\hline Educación & $\begin{array}{c}\mathbf{0 . 0 5 1 9 * * *} \\
(0.0101)\end{array}$ & $\begin{array}{l}\mathbf{0 . 0 4 8 1} * * * \\
(0.00993)\end{array}$ & $\begin{array}{c}\mathbf{0 . 0 4 6 8 * * *} \\
(0.00986)\end{array}$ & $\begin{array}{l}\mathbf{0 . 0 5 1 0}^{* * *} \\
(0.00974)\end{array}$ & $\begin{array}{l}\mathbf{0 . 0 5 1 1 * * *} \\
(0.00972)\end{array}$ \\
\hline Experiencia & $\begin{array}{c}0.0104 \\
(0.00918)\end{array}$ & $\begin{array}{c}0.00740 \\
(0.00901)\end{array}$ & $\begin{array}{c}0.00787 \\
(0.00893)\end{array}$ & $\begin{array}{c}0.0175 * \\
(0.00900)\end{array}$ & $\begin{array}{l}0.0183 * * \\
(0.00900)\end{array}$ \\
\hline Experiencia $^{2}$ & $\begin{array}{l}-0.000242 \\
(0.000150)\end{array}$ & $\begin{array}{l}-0.000159 \\
(0.000148)\end{array}$ & $\begin{array}{l}-0.000191 \\
(0.000147)\end{array}$ & $\begin{array}{c}-0.000296 * * \\
(0.000146)\end{array}$ & $\begin{array}{c}-0.000311^{*} * \\
(0.000146)\end{array}$ \\
\hline Ocupado & & $\begin{array}{c}0.339 * * * \\
(0.0621)\end{array}$ & $\begin{array}{l}0.326 * * * \\
(0.0617)\end{array}$ & $\begin{array}{c}0.309 * * * \\
(0.0608)\end{array}$ & $\begin{array}{c}0.311 * * * \\
(0.0607)\end{array}$ \\
\hline Casado & & & $\begin{array}{l}0.267 * * * \\
(0.0765)\end{array}$ & $\begin{array}{l}0.182 * * \\
(0.0772)\end{array}$ & $\begin{array}{l}0.179 * * \\
(0.0771)\end{array}$ \\
\hline Mujer & & & & $\begin{array}{c}-0.386 * * * \\
(0.0789)\end{array}$ & $\begin{array}{c}-0.379 * * * \\
(0.0788)\end{array}$ \\
\hline Pasto & & & & & $\begin{array}{l}-0.0959^{*} \\
(0.0524)\end{array}$ \\
\hline Constante & $\begin{array}{c}12.53 * * * \\
(0.188)\end{array}$ & $\begin{array}{c}12.30^{* * * *} * \\
(0.189)\end{array}$ & $\begin{array}{c}12.19 * * * \\
(0.190)\end{array}$ & $\begin{array}{c}12.24 * * * \\
(0.188)\end{array}$ & $\begin{array}{c}12.31 * * * \\
(0.191)\end{array}$ \\
\hline $\begin{array}{l}\text { Breusch - Pagan } \\
\text { Chi } 2 \text { (1) } \\
\text { P- Valor }\end{array}$ & & Test: & $\operatorname{Var}(\mathrm{u})=0$ & & $\begin{array}{l}132,01 \\
0,0000\end{array}$ \\
\hline $\begin{array}{l}\text { Hausman Test } \\
\text { Chi } 2(7) \\
P-\text { Valor }\end{array}$ & Test: Ho: & difference in & coefficients & ot systematic & $\begin{array}{c}97,39 \\
0,0000\end{array}$ \\
\hline Observaciones & 1,594 & 1,594 & 1,594 & 1,594 & 1,594 \\
\hline $\begin{array}{l}R^{2} \\
\text { Individuos }\end{array}$ & $\begin{array}{c}5,9 \% \\
911\end{array}$ & $\begin{array}{c}9,9 \% \\
911\end{array}$ & $\begin{array}{c}11,5 \% \\
911\end{array}$ & $\begin{array}{c}14,5 \% \\
911\end{array}$ & $\begin{array}{c}14,9 \% \\
911\end{array}$ \\
\hline
\end{tabular}

Fuente: Esta Investigación. Standard errors in parentheses ${ }^{* * *} p<0.01,{ }^{* *} p<0.05,{ }^{*} p<0.1$

Los datos indican que de manera significativa estadísticamente, un año más de educación incrementa aproximadamente en 5,11\% el ingreso de los pastusos. Este 
resultado es prácticamente invariable ante diferentes controles como ser casado, ser oriundo de Pasto, estar ocupado o pertenecer al género femenino. Cuando los datos se analizan por niveles de estudio, se encuentra que tener primaria completa reduce en aproximadamente un 12,3\% el salario promedio mensual de las personas, mientras que tener estudios profesionales de pregrado o postgrado lo incrementa en un $34,7 \%$. Asimismo, los datos reafirman la brecha salarial entre hombres y mujeres, dónde éstas devengan un 37,9\% menos que los hombres, ratificando los datos de la Red ORMET en el 2013.

Para analizar estos datos en contexto, se encuentra que apenas el 5\% de la población cuenta con salarios mensuales superiores a $\$ 2.400 .000$, mientras que el $50 \%$ recibe salarios mensuales inferiores a los $\$ 515.000$. Asimismo, solo un $10 \%$ de la población cuenta con educación superior de pregrado o postgrado, cuando el 50\% de la población tiene estudios de bachillerato o inferiores.

La importancia de la educación para los pobladores de Pasto queda en evidencia por su influencia sobre el nivel de ingresos salariales pero, además, los datos permiten establecer su relevancia en las probabilidades de obtener empleo, lo datos son los siguientes:

Tabla 5. Educación y Empleo en Pasto, 2008 - 2010

\begin{tabular}{l|ccc}
\hline \multirow{2}{*}{ VARIABLES } & \multicolumn{3}{|c}{ Ocupado - Modelo de Probabilidad Lineal } \\
\cline { 2 - 4 } educación & $(\mathbf{1})$ & $(\mathbf{2})$ & $(\mathbf{3})$ \\
& $\mathbf{0 . 0 3 6 8} \mathbf{8}^{* * *}$ & $\mathbf{0 . 0 3 4 4} * * *$ & $\mathbf{0 . 0 3 4 8} * * *$ \\
experiencia & $(0.00166)$ & $(0.00166)$ & $(0.00166)$ \\
& $0.00507^{* * *}$ & $0.00343^{* * *}$ & $0.00363^{* * *}$ \\
casado & $(0.000407)$ & $(0.000452)$ & $(0.000451)$ \\
& & $0.141 * * *$ & $0.130^{* * * *}$ \\
mujer & & $(0.0177)$ & $(0.0177)$ \\
& & & $-0.0951^{* * *}$ \\
Constante & & & $(0.0151)$ \\
& $-0.0714^{* * *}$ & $-0.0709 * * *$ & -0.0230 \\
Observaciones & $(0.0209)$ & $(0.0206)$ & $(0.0219)$ \\
Individuos & 4,219 & 4,219 & 4,219 \\
$\mathrm{R}^{2}$ & 1,784 & 1,784 & 1,784 \\
\hline
\end{tabular}

Fuente: Esta Investigación. Standard errors in parentheses ${ }^{* *} p<0.01,{ }^{* *} p<0.05,{ }^{*} p<0.1$

Mediante estimación por Modelo de Probabilidad Lineal, se puede establecer que los años de educación resultan altamente significativos, estadísticamente hablando, a la hora de determinar la probabilidad de encontrar empleo en el municipio de Pasto, pues un año adicional de educación aumenta en 3,5\% la probabilidad de estar ocupado. Igualmente, la experiencia laboral, estar casado y ser mujer también resultan ser significativas. Sobre este último factor se encuentra que ser mujer reduce en $9,5 \%$ las probabilidades de encontrar empleo en la ciudad aun cuando tengan mayor educación y experiencia laboral promedio que los hombres. Queda establecido entonces que la educación en Pasto además de proporcionar mayores ingresos, también incrementa la posibilidad de conseguir empleo. 


\section{Conclusiones}

La economía de Pasto, aunque volátil ha mantenido un crecimiento sostenido por encima del promedio nacional y departamental. Sin embargo, su aporte al PIB nacional no supera el 1\%, aun cuando aporte más del 53\% al PIB departamental, evidenciando un municipio líder de un departamento relegado del desarrollo nacional, al que la educación por ser un agente que posibilita el cambio estructural de las sociedades, puede ayudarle a superar esta realidad. No obstante, el menor pago educacional con relación al país, limita su aporte.

Si bien las personas de género masculino del municipio de Pasto tienen mayores ingresos que las mujeres, éstas registran mayores niveles de educación y experiencia laboral promedio. En términos de procedencia, las personas oriundas de Pasto registran menores años de escolaridad y experiencia laboral que los foráneos y por ende son retribuidos con menores ingresos. En consecuencia, pertenecer al género femenino y/o ser oriundo de Pasto puede constituirse en un hándicap en el mercado laboral de este municipio.

A través de la estimación del modelo de Mincer mediante el método de Efectos Fijos en datos panel, y una vez contrastada su validez y superada la presencia de heterogeneidad no observada en el término de error y de endogeneidad, no se rechaza la hipótesis de que existe una relación positiva entre el ingreso y la educación en el municipio de Pasto. Este resultado permite afirmar que un año adicional de educación incrementa aproximadamente en 5,11\% el ingreso de los pastusos. Este valor es un tanto inferior a resultados ofrecidos por similares investigaciones en ciudades grandes o pequeñas, tal como se planteó el argumento central.

Esta investigación también pudo establecer, mediante estimación por Modelo de Probabilidad Lineal, que los años de educación resultan altamente significativos, estadísticamente hablando, a la hora de determinar la probabilidad de encontrar empleo en el municipio de Pasto, pues un año adicional de educación aumenta en 3,5\% la probabilidad de estar ocupado. Así mismo, la experiencia laboral, estar casado y ser mujer también resultan ser significativas. 


\section{Referencias}

1. ARIAS, Yesid y CHAVES, Álvaro (2002). Cálculo de la tasa interna de retorno de la educación en Colombia. En: Documento de Trabajo, No. 2, Universidad Externado de Colombia, Bogotá.

2. ARROW, K. (1972). Some Mathematical Models of Race Discrimination in the Labor Market en Racial Discrimination in Economic Life, Pascal, A. (ed.), Lexintong: D.C. Heath, pp. 187-203.

3. BARRERO, Andrea; VÉLEZ, Clara; LÓPEZ, Iván; ROSALES, Ramón y MOGOLLÓN, Darwin (2004). Evaluación económica del componente educativo del Programa Familias en Acción del Plan Colombia, En: Documento CEDE, No. 2004-39.

4. BECKER, Gary (1964). Human Capital. 2a. ed., National Bureau Economics Research. Columbia University Press. Nueva York.

5. BLAUG, M. (1970). An Introduction to the Economics of Education. Allen Lane, ThePenguinPress. Londres.

6. CARNOY, Martin (1977). Economía y educación. Chicago: University of Chicago Press. Traducción y actualización por: BENITEZ ROCHEL, J.J. y ROBLES TEIGEIRO, L. pp. 20 y 21.

7. CASAS, Andrés; SEPÚLVEDA, Carlos y GALLEGO, Juan (2003). Retornos de la educación y sesgo de habilidad: teoría y aplicaciones en Colombia. En: Lecturas de Economía, No. 58.

8. CASTELLAR, C. y URIBE, J. (2001). Una Aproximación Econométrica a la Tasa de Retorno Social de la Educación. En: Sociedad y Economía. Facultad de Ciencias Sociales y Económicas, CIDSE, Universidad del Valle, Cali.

9. CEDRE - Centro de Estudios de Desarrollo Regional y Empresarial (2011). Cuentas Económicas del municipio de Pasto. 2005-2010. Facultad de Ciencias Económicas y Administrativas, Universidad de Nariño. San Juan de Pasto.

10. EIJS, Patrick Van y HEIJKE, Hans (1996). The relation between the wage, job-related training and the quality of the match between occupations and types of education, ROA-RM-1996/6E, Maastricht, Research Centre for Education and the Labour Market, pp. 1-57.

11. FORERO, Nohora Y. y GAMBOA, Luis Fernando (2007). Cambios en los Retornos de la Educación en Bogotá entre 1997 y 2003. En: Lecturas de Economía, no. 66. Universidad de Antioquia, Medellín.

12. FREIRE SEOANE, María Jesús y TEJEIRO ÁLVAREZ, Mercedes (2011). Las ecuaciones de Mincery las tasas de rendimiento de la educación en Galicia. Observatorio Ocupacional de A Coruña, España.

13. GAREN, John (1984). The Returnsto Schooling: A Selectivity Bias Approachwith a Continous Choice Variable. En: Econométrica, vol. 52, núm. 5, septiembre.

14. GONZÁLEZ, Iván (1982). Tasa de rendimiento de la educación, productividad y nivel de pobreza en la pequeña industria. En: Revista Desarrollo y Sociedad. No. 7.

15. GRILICHES, Zvi (1977). Estimating the Returns to Schooling: Some Econometric Problems. En: Econométrica, vol. 45, núm. 1, enero.

16. HALEY, William J. (1976). Estimation of the Earnings Profile from Optimal Human Capital Accumulation. En: Econométrica, vol. 44, núm. 6, noviembre.

17. HECKMAN, James (1979). Sample selection bias as a specification error. En: Econométrica, Vol. 47, №. 1. 
18. JOHNSON, Harry (1975). On Economics and Society. University of Chicago Press, Chicago.

19. JOHNSON, Thomas (1980). Returns from Investment in Human Capital. En: American Economic Review.

20. KENNY, L.; LEE, L.; MADDALA, G.S.; y TROST, R.P. (1979). Returns to College Education: An Investigation of Self-Selection Bias Based on the Project Talent Data. En: International Economic Review, Vol. 20, número 3.

21. KUGLER, Bernardo y REYES, Álvaro (1975). Educación y mercado de trabajo urbano en Colombia: una comparación entre sectores modernos y no modernos. CCRP.

22. LAU, L.; JAMISON, D.; LIU, S.y RIVKIN, S. (1993). Education and Economic Growth, Some Cross-Sectional Evidence from Brazil. En: Journal of Development Economics, Vol. 41.

23. LÓPEZ MOGUEL, María del Rosario (2009). Efectos de la correspondencia entre educación y empleo de los jóvenes en el mercado de trabajo mexicano. [Artículo en Internet].<http://www.scielo.org.mx/ scielo.php?pid=S140566662009000300012\&script=sci_arttext >. [Consultado: 13 de julio de 2014].

24. MINCER, Jacob (1974). Schooling Experience and Earnings. National Bureau Economics Research. Columbia University Press. Nueva York.

25. MORA, James (2003). Sheepskin Effects and Screening in Colombia. Colombian Economic Journal, No. 1.

26. NÚÑEZ, Jairo y SÁNCHEZ, Fabio (1998). Educación y salarios relativos en Colombia: 1976-1995. Determinantes, evolución e implicaciones para la distribución del ingreso. En: Archivos de Macroeconomía, No. 74, Departamento Nacional de Planeación, Bogotá.

27. NÚÑEZ, Jairo y SÁNCHEZ, Fabio (2003). A Dynamic Analysis of Human Capital, Female Work-Force Participation, Returns to Education and Changes in Household Structure in Urban Colombia, 1976-1998. Colombian Economic Journal, No. 1.

28. ORMET. Red de Observatorios del Mercado de Trabajo (2012). Diagnóstico socioeconómico y del mercado de trabajo Ciudad de Pasto. PNUD Oficina Colombia. San Juan de Pasto.

29. ORMET. Red de Observatorios del Mercado de Trabajo (2013). Brechas de género en el mercado de trabajo de Pasto. PNUD Oficina Colombia. San Juan de Pasto.

30 PERFETTI, Mauricio (1996). Diferencias salariales entre hombres y mujeres no asalariadas durante el período 1984-1994. En: Revista Planeación y Desarrollo, No. 27. Departamento Nacional de Planeación, Bogotá.

31. PRADA, Carlos F. (2006). ¿Es rentable la decisión de estudiar en Colombia? En: Ensayos de Política Económica, No. 51.

32. PSACHAROPOULOS, George y VÉLEZ, E. (1992). Schooling, Ability, and Earnings in Colombia: 1988. En: Economic Development and Cultural Change, Vol. 40.

33. QUIÑÓNEZ DOMÍNGUEZ, Mauricio y RODRÍGUEZ SINISTERRA, Juan Antonio (2010)."Rendimiento de la Educación en las Regiones Colombianas, un análisis usando la descomposición Oaxaca-Blinder". En: CIDSE- Universidad del Valle. Documento de Trabajo No. 128. Santiago de Cali.

34. RAYMOND, Byron y MANALOTO, Evelyn (1990). Returns to Education in China. En: Economic Development and Cultural Change, Vol. 38.

35. RIASCOS H., Julio César y ERAZO Iván Fredy (2010). Situación del mercado laboral de los profesionales en San Juan de Pasto: un análisis de modelación micro-econométrica con datos de corte transversal. En: Revista Tendencias Vol. XII. No. 1 1er. Semestre 2011, pp. 44-91.

36. RIASCOS H., Julio César (2011). Situación laboral de la mujer profesional en pasto, 2010: Un análisis de modelación micro-econométrica. En: Revista Tendencias Vol. XII. No. 2 2do. Semestre 2011, pp. 9-34. 
37. ROJAS, Mariano; ANGULO, Humberto y VELÁZQUEZ, Irene (2000). Rentabilidad de la inversión en capital humano en México. En: Economía Mexicana. Nueva Época, Vol. IX, núm. 2, segundo semestre.

38. ROSALES ÁlVAREZ, Ramón Antonio, et. al. (2013). Fundamentos de Econometría, Teoría y Aplicaciones. Universidad de los Andes. Enero, Bogotá.

39. ROSEN, Sherwin (1977). Human Capital: A Survey of Empirical Research, en R.G. Ehrenberg (ed.). En: Research in Labor Economics, Vol. 1, Greenwich, JAI Press.

40. ROSS, Westerfield (1995). Finanzas Corporativas. Irwin.

41. SALAS, M. (2004). Rendimientos Privados de la Inversión en Educación Superior a partir de Ecuaciones de Ingresos. En: Revista de Economía Pública 169-(2/2004). Hacienda Pública Española, Instituto de Estudios Fiscales.

42. SAPELLI, C. (2009). Los Retornos a la Educación en Chile: Estimaciones por Corte Transversal y por Cohortes. En: Documento de trabajo 349, Instituto de Economía, Pontificia Universidad Católica de Chile. Santiago.

43. SCHULTZ, T. (1968). Return of Education in Bogotá, Colombia. Rand Corporation.

44. SELOWSKY, M. (1968). The Effect of Unemployment Labour-Market Segmentation and Returns oEducated: The Case of Colombia. Center for International Affairs. Harvard University. Publicado en español. En: Revista de Planeación y Desarrollo. Vol. 1, julio de 1969, Bogotá.

45. TENJO, Jaime (1993a). Evolución de los Retornos de la Inversión en Educación 1976-1989. En: Revista de Planeación y Desarrollo. Vol. XXIV. Diciembre, Bogotá.

46. TENJO, Jaime (1993b). Educación, Habilidad, Conocimientos e Ingreso. En: Planeación y Desarrollo. Vol. XXIV. Diciembre, Bogotá.

47. TENJO, Jaime y BERNAT, Luisa Fernanda (2002). Evolución de las diferencias salariales entre hombres y mujeres: comparación de seis países latinoamericanos, mimeo.

48. VELÁSQUEZ, Clara (2001). Análisis empírico de las diferencias salariales entre hombres y mujeres en Colombia. Universidad del País Vasco.

49. ZÁRATE, Héctor (2003). Cambios en la estructura salarial: Una historia desde la Regresión cuartílica En: Borradores de Economía, No. 245, Banco de la República, Bogotá. 